\title{
ANÁLISIS DE LOS FLUJOS EXTREMOS DE ELECTRONES ENERGÉTICOS EN EL CINTURÓN DE RADIACIÓN EXTERIOR Y EN LA ANOMALÍA MAGNÉTICA DEL ATLÁNTICO SUR
}

\author{
Vanina Lanabere $^{1}$ y Sergio Dasso ${ }^{1,2,3}$ \\ ${ }^{1}$ Universidad de Buenos Aires, Facultad de Ciencias Exactas y Naturales, Departamento de Ciencias \\ de la Atmósfera y los Océanos, Buenos Aires, Argentina \\ ${ }^{2}$ CONICET-Universidad de Buenos aires, Instituto de Astronomía y Física del Espacio, Buenos \\ Aires, Argentina \\ ${ }^{3}$ Universidad de Buenos Aires, Facultad de Ciencias Exactas y Naturales, Departamento de Física, \\ Buenos Aires, Argentina
}

(Manuscrito recibido el 28 de mayo de 2020, en su versión final el 31 de agosto de 2020)

\section{RESUMEN}

Los cinturones de radiación de van Allen son regiones en el entorno espacial terrestre que presentan iones y electrones energéticos atrapados por el campo geomagnético. El incremento del flujo para estas partículas energéticas durante tormentas geomagnéticas tiene un gran interés para la meteorología del espacio, debido principalmente al impacto que tiene sobre los satélites y la actividad espacial humana. Un entendimiento detallado de los flujos extremos alcanzados por electrones a diferentes energías, así como la frecuencia de ocurrencia es esencial para el diseño específico de satélites y para el desarrollo de tecnologías satelitales.El objetivo principal de este trabajo es estudiar los flujos extremos de electrones en los cinturones de radiación terrestre, para un rango de energías entre $0,249 \mathrm{MeV}$ y $0,802 \mathrm{MeV}$ a $660 \mathrm{~km}$ de altitud sobre la superficie de la Tierra, usando mediciones realizadas por el detector ICARE-NG/Carmen-1 a bordo del satélite polar argentino SAC-D. Un estudio estadístico basado en la teoría de valores extremos se ha implementado al promedio diario del flujo de electrones en el cinturón de radiación exterior y en la Anomalía Magnética del Atlántico Sur (AMAS).Encontramos que la función de distribución acumulada del promedio diario del flujo de electrones parece tener un límite superior finito en el centro del cinturón de radiación exterior $(4,0<L<4,5)$ y para electrones con energías entre $E>0,270 \mathrm{MeV}$ y $E>0,413$ $\mathrm{MeV}$. El flujo de electrones extremo esperado en tiempos de 10, 50 y 100 años fueron calculados para $L=4,5$ mostrando, en general, una tendencia a disminuir mientras aumenta la energía. A pesar de que los resultados en la AMAS sugieren que la función de distribución acumulada del flujo de electrones no tiene un límite superior finito, no es posible concluir con certeza este resultado por no tener significancia estadística. Los resultados presentados en este trabajo son importantes para los ingenieros de satélites, de cara a mejorar dispositivos y materiales para el desarrollo de los futuros satélites. También, la magnitud esperada de un evento extremo en el cinturón de radiación exterior es de interés para las aseguradoras satelitales de cara a evaluar potenciales escenarios de desastres. 
Palabras clave: Meteorología del Espacio, Cinturones de Radiación, Teoría de valores extremos.

\title{
ANALYSIS OF EXTREME ENERGETIC ELECTRON FLUXES IN THE OUTER RADIATION BELT AND SOUTH ATLANTIC MAGNETIC ANOMALY
}

\begin{abstract}
The van Allen radiation belts are regions in the terrestrial space environment that present energetic ions and electrons trapped by the geomagnetic field. The increase of fluxes for these energetic particles during geomagnetic storms has a major interest for Space Weather, mainly due to the impact on satellites and human activities in space. A detailed knowledge of the extreme fluxes reached for different electron energies as well as the frequencies of occurrence is essential for the specific design of satellites and for the development of satellite technologies. The main purpose of the present work is to study the extreme electron fluxes in the terrestrial radiation belts, for an energy range between $0.249 \mathrm{MeV}$ and $0.802 \mathrm{MeV}$ at $660 \mathrm{~km}$ of altitude above the Earth surface, using measurements made by the detector ICARE-NG/Carmen-1 on board the polar Argentinean satellite SAC-D. A statistical analysis based on the extreme value theory was implemented for the daily average electron flux in the outer radiation belt and in the South Atlantic Magnetic Anomaly (SAMA).We found that the cumulative distribution function of the daily averaged electron flux is likely to have a finite upper limit in the core of the outer radiation belt $(4.0<$ $L<4.5)$ and for electron energies between $E>0.270 \mathrm{MeV}$ and $E>0.413 \mathrm{MeV}$. The extreme electron flux value expected in 1, 10, 50 and 100 years were computed at $L=4.5$, showing a general decreasing trend with increasing energy. Although the results in the SAMA suggest that the cumulative distribution function of the electron flux is likely to not have a finite upper limit, this result is not statistically significant.The results presented in this work are important for the satellite engineers to improve devices and materials for the development of future satellites. Also, the likely magnitude of an extreme event in the outer radiation belt is of interest to the satellite insurers to help them evaluate potential disaster scenarios.
\end{abstract}

Keywords: Space weather, Radiation belts, Extreme value theory.

\section{INTRODUCCIÓN}

Space Weather events produce disturbances in the Earth environment that can affect space and ground-based technologies. It is now well understood that Space Weather represents a significant threat on navigation, communications and human-health in space. Different economic sectors are more or less affected depending on the technology associated, the time of exposure and the strength of the event.
International institutions, as for instance the World Meteorological Organization (WMO), the International Civil Aviation Organization (ICAO), the United Nations Office for Outer Space Affairs (UNOOSA), have begun to develop programs and activities on Space Weather, some of them with the aim of having answers to the negative effects of extreme Space Weather events.

However, one of the main open questions to become aware of the seriousness of these risks 
is how frequent the most extreme events are. The study of the behaviour of the tail of the distribution function (TDF) of some critical physical quantities associated with extreme events can help to get closer to this answer. For instance, to study the TDF of the flux of energetic particles at given regions in space is of major interest for the specific design of satellites and for the development of modern technologies (e.g. Ruzmaikin et al., 2011; Elvidge and Angling,2018).

The van Allen radiation belts are regions in the terrestrial space environment that present energetic ions and electrons trapped by the geomagnetic (e.g., Prölss, 2012). As the motion of these particles follows the magnetic field lines, it is useful to define the $\mathrm{L}$ parameter, which describes the distance where a magnetic field line crosses the Earth's magnetic equator plane, defined only for an aligned magnetic dipole field (MCIlwain, 1961).There exists mainly two zones, the inner radiation belt that extends from $L \sim 1.2-2.5$ (i.e. the magnetic field lines which cross the Earth's magnetic equator from 1.2 Earth-radii to 2.5 Earth-radii) and presents a maximum flux of high-energy protons at $L=1.5$ and, the outer radiation belt that extends from $L \sim 3.0-8.0$ with its maximum flux of energetic electrons located near $L=3.5$ (Walt, 2005). Between these two zones there is a region, called slot region, with relative absence of energetic particles during quiet periods.

During a significant perturbation of the geospace and the upper atmosphere, the population of energetic particles in the radiation belts is perturbed. Although the inner radiation belt keeps almost stable, the outer radiation belt populations present large variability, principally its size and location can change dramatically.For instance, Reeves et al. (2003) found that geomagnetic storms can either increase or decrease the fluxes of relativistic electrons in the radiation belts, and that only during about the half of all storms the fluxes of relativistic electrons increased. Meanwhile, Xiong et al. (2015) found that storms preferentially enhance the electron fluxes at energies between $0.3-2.5$ $\mathrm{MeV}$.

The energetic particles in the radiation belts can impact satellites, creating a number of hazards to their operation and longevity. The specific effects and impacts will depend upon satellite orbit and on the fluxes of different particle energies.Electrons with energies of $100 \mathrm{keV}$ interact with surface materials of the spacecraft leading to surface charging. As a result, electrostatic potential differences can arise between different surfaces of the spacecraft, leading to an electrostatic discharge which can damage the surface materials of the satellite (Koons and Fennell, 2006). Larger energy electrons, of a few $\mathrm{MeV}$, can penetrate into the outer shield of the spacecraft and deposit charge inside insulating materials. Thus, the internal electrostatic discharge occurs very close to vulnerable devices. As a result, the spacecraft can experience permanent damage to the dielectric, component failure, phantom commands causing uncontrolled behaviour of the spacecraft (Wrenn et al., 2002), and other undesirable effects.

Since the geomagnetic field in the South Atlantic Magnetic Anomaly (SAMA) is relatively weakest over the western South Atlantic Ocean and part of South America, trapped particles of the radiation belts approach closer to the Earth surface which leads to a deeper penetration of energetic particles into the ionosphere.Sheldon and Benbrook (2004) found that the smaller strength of the surface geomagnetic field in the southern hemisphere (the SAMA) dictates that steady-state precipitation of trapped electrons occurs there. As the electron flux in the outer radiation belt significantly increase during a geomagnetic storm, also it was observed an enhancement in the energetic electron precipitation in the SAMA. For example, Nishino et al. (2002) have noted absorption of cosmic radio noise in the ionosphere due to electron precipitation into the SAMA ionosphere specially during the main and recovery phases of a magnetic storm.Abdu 
et al. (1981) found ionisation enhancements associated with magnetic storms due to particle precipitation of high-energy charged particles in the South Atlantic magnetic anomaly. Horne et al. (2009) found that for the outer radiation belt, electron precipitation for $E>300 \mathrm{keV}$ peaks during the main phase of storms whereas that $E>1 \mathrm{MeV}$ peaks can be present during the recovery phase. Precipitation of electrons with $E>300 \mathrm{keV}$ can occur at all geographic longitudes in both hemispheres whereas that for $E>1 \mathrm{MeV}$ occurs mainly poleward of the SAMA region.

Particle precipitations in the SAMA region could lead to impulsive pulsations observed in the horizontal component of the geomagnetic field near the centre of the SAMA (Trivedi et al., 2005). Furthermore, these perturbation in the geomagnetic field may contribute to the Geomagnetically induced currents (GICs) production (Caraballo et al., 2013).These GICs may disturb the operation of power systems, cause damage to power transformers, and even result in power blackouts (deVilliers et al., 2016).

The low and high energetic electron fluxes in the outer radiation belt are the source of many of the technological hazards for Low, Medium, and Geosynchronous Earth Orbiting (LEO, MEO, and GEO) spacecraft. Especially during a geomagnetic storm there is higher risk of damage at all times the spacecraft passes through the SAMA (Heirtzler et al., 2002). Also ground power systems can be affected like transformers (resulting in power blackouts) and underground pipelines (resulting in degradation of their transport systems). Thus, to have knowledge about extreme fluxes of energetic electrons and the possible return time of the maximum events is a key goal to the development of new satellite and ground system technologies.

The extreme value analysis has been used for many studies of extreme events in meteorology (e.g., Re and Barros, 2009; Tencer and
Rusticucci, 2012) and for extreme events in Space Weather. For example, it was applied to X-ray flux (Elvidge and Angling, 2018), or to solar energetic proton fluxes (Ruzmaikin et al., 2011). In particular,the extreme value analysis of energetic electron flux in the radiation belt were done by Koons (2001) and Meredith et al. (2015). They studied daily electron fluxes with energies larger than $2 \mathrm{MeV}$ with GOES satellite, (i.e. at a fixed value of $L \sim 6.6$ ) and using the peaks over threshold (POT) method. Other analyses were done by O'Brien et al. (2007)and Meredith et al. (2016). They used the Maximum of Blocks method and extended the energies levels of electrons between some $\mathrm{keV}-\mathrm{MeV}$, also they used data from highly elliptical and low Earth orbit, respectively to extend the study to $L \sim 3-8$.

In this work, we explore the extreme electron fluxes with energies in the range of $0.249 \mathrm{MeV}$ to $0.802 \mathrm{MeV}$ measured with the particle detector ICARE-NG on board the Argentinean polar orbit satellite SAC-D at $660 \mathrm{~km}$ altitude.We applied the POT method in the outer radiation belt $(L=3.5-5.0)$ andin the South Atlantic Magnetic Anomaly.In the Methodology section, the extreme value analysis used to study the extreme electron fluxes in the outer radiation belt and in the South Atlantic Magnetic Anomaly is described. The method applied to data in both regions is described in Data section. The Results section presents the shape parameters that describe the distribution tails behaviour and the return levels for both regions. Finally, we present the conclusions of this work.

\section{METHODOLOGY}

In this work we performed a statistical technique used for modelling and estimating of the distribution tail behaviour known as an extreme value analysis, (i.e. Coles, 2013). There are two well-known general characterizations for the extreme value. One is based on the maximum of blocks, the other is based on exceedances/peaks of a high threshold (POT). For the POT method the raw data consist 
of a sequence of independent and identically distributed measurements $x_{1}, \ldots, x_{n}$. Extreme events are identified by defining a high threshold $\mathrm{u}$, for which the exceedances are $x_{i}: x_{i}>$ $u$. Label these exceedances by $x(1), \ldots, x(k)$, define threshold excesses by $y_{j}=x_{(j)}-u$, for $j=1, \ldots, k$. The $y_{j}$ may be regarded as independent realizations of a random variable whose distribution can be approximated by a member of the generalized Pareto family. In the case of the POT method, the appropriate function to fit the cumulative probability density function of extreme events is the Generalized Pareto (GP) distribution (Pickands, 1975) defined by,

$G_{k, \mu, \sigma}(X)= \begin{cases}1-\left(1+\frac{k(X-\mu)}{\sigma}\right)^{-\frac{1}{\kappa}}, & \text { for } k \neq 0 \\ 1-\exp \left(-\frac{X-\mu}{\sigma}\right) & , \text { for } k=0\end{cases}$

where $X$ is the random variable associated with the electron flux, $\mu$ and $\sigma$ are the location and scale parameters, respectively. The shape parameter, $k$, describes the behaviour for extreme values of the distribution. The GP distribution has three basic forms depending on the value of the shape parameter: i) distributions whose tails decrease exponentially, such as the normal distribution, lead to a GP shape parameter of zero, ii) distributions whose tails decrease as a polynomial, such as Student's $\mathrm{t}$, lead to a positive shape parameter and iii) distributions whose tails are finite, such as the beta functions, lead to a negative shape parameter (Coles, 2013). By definition, the GP distribution models exceedances above a threshold. In particular, the GP distribution function $(G(x))$ is a suited candidate to represent the probability that a random variable $X$ exceeds some value $x$ given that it already exceeds a threshold $\mu$,

$$
P(X>x \mid X>u)=1-G(x)
$$

The $X_{N}$ return level is the level expected to be exceeded once every $N$ years, defined by Coles (2013) as:

$$
X_{N}=u+\frac{\sigma}{k}\left(\left(N n_{d} n_{c} / n_{t o t}\right)^{k}-1\right), \quad \text { for } k \neq 0
$$

where $N$ is the number of years expected to wait in order to get a $X_{N}$ value, nd is the number of observations per year, $n_{c}$ is the number of observations exceeding the threshold and ntotthe total number of data points.

\section{DATA}

The particle detector ICARE-NG on board the polar orbit satellite SAC-D provides information about the Omni-directional Integral Electron Flux (FEIO) for a width range of $L$ values (i.e. $L=1-8$ ) and a wide range of energy (0.249 to $1.192 \mathrm{MeV}$ ) divided in 19 energy channels with a temporal cadence of 16 seconds during the period from August/2011 to June/2015 that corresponds to the maximum phase of the solar cycle 24. ICARE-NG is the new generation of the particle detector ICARE on board the Argentinean satellite SAC-C. Furthermore, ICARE-NG was also on board during the JASON-2 mission, and on JASON-3. A complete description of the ICARE-NG/CARMEN-1 instrument can be found in Boscher et al. (2011). Protons with energies above $100 \mathrm{MeV}$ usually cannot be shielded by solid-state detectors and may contaminate the electron observations (Vampola, 1998). This contamination by energetic protons has been studied to affect several spacecrafts, for example in the Van Allen probe MagEIS and in the Cluster RAPID/IES (see Claudepierre et al., 2015; Smirnov et al., 2019, respectively). This contamination is also observed in ICARE-NG data (Boscher et al., 2014). Since the electron fluxes data have been contaminated by protons fluxes during solar proton events (SPE), the data was carefully examined to detect SPE periods. A day is considered to be affected by a SPE if the electron flux for each energy channel at $L=7-7.25$ excess in 2 standard deviation the mean value at $L=7-7.25$. We cross-checked those days with the SPE list documented by NOAA (ftp://ftp.swpc.noaa.gov/pub/ indices/SPE.txt) and found that all the days that excess in 2 standard deviation the mean value at $L=7-7.25$ were in the NOAA 
SPE list. Finally, we removed from the data all the days that were affected by a SPE.This procedure is followed in order to remove SEPs events that have a significant effect on the electron flux measurements.

The daily averaged electron flux for energies $E>0.270 \mathrm{MeV}$ and $E>0.802 \mathrm{MeV}$ for the period August/2011 to June/2015 and the $K_{p}$ index for the same period are shown in Fig. 1a,b.Before removing the SPE periods, as described in Section 3, the SPE can be seen in Fig. 1a,b as red vertical lines that extends from $L \sim 3-8$. For instance, the X-class solar flare detected on March 72012 produced a large SPE.The outer radiation belt extends from $L=3$ to $L=7$ with a maximum around $L=3.5-5.0$ and the inner radiation belt can be observed at $L=1.5-2.0$. The outer radiation belt presents several fluctuations along time with enhancements of almost two order magnitude in a few days. These sudden increases are well known, and they are associated with geomagnetic storms. In contrast with the outer radiation belt, the inner belt stays almost constant.

The $K_{p}$ index for the same period is shown Fig. 1c, as a measurement of the magnitude of geomagnetic disturbance on a planetary scale. It ranges from 0 to 9 , with zero being very quiet and 9 indicating an extreme geomagnetic storm (Bartels, 1949). This data is available at https: //cdaweb.gsfc.nasa.gov/index.html/.It can be seen that the sudden increases of electron fluxes in the outer radiation belt correspond to $K_{p}$ values larger than $K_{p}=5$ (i.e, during geomagnetic storms). Also, during these events an enhancement of the electron flux is observed in the slot region (i.e. $L \sim 3$ ) after the most intense geomagnetic storms (i.e. $K p>5$ ).

The electron flux enhancement in the core of the outer belt shown in Fig. 1a is still evident in Fig. 1b. The electron flux presents an enhancement of two order magnitude during the most intense geomagnetic storms. However, while increasing the energy channel, the electron flux enhancement is confined in a more stretch region $L=3$ to $L=6$, the outer bound of the outer radiation belt is reduced, and the slot region almost do not present any perturbation.

The temporal mean value from August/2011 to June/2015 of all the data set in geographical coordinates and spatial resolution of $5^{\circ} \times 5^{\circ}$ and $E>0.270 \mathrm{MeV}$ is shown in Fig. 2a. There is a maximum of electron flux $>10^{5} \mathrm{~cm}^{-2} \mathrm{~s}^{-1} \mathrm{sr}^{-1}$ in the region of the SAMA that extends round south America and South Atlantic Ocean. A second relative maximum is observed in high latitudes near the auroral zone, these electron population are associated with $L>2$ and correspond to the electron particles in the outer radiation belt with a mean value of $>10^{4}$ $\mathrm{cm}^{-2} \mathrm{~s}^{-1} \mathrm{sr}^{-1}$. The solid line in Fig. 2a represents the core of the SAMA defined for each energy channel as the region that exceeds the 98th percentile of the 2011-2015 mean value in the same energy channel (e.g., for $E>0.270 \mathrm{MeV}$ is $\left.1.1 \times 10^{5} \mathrm{~cm}^{-2} \mathrm{~s}^{-1} \mathrm{sr}^{-1}\right)$.

The geomagnetic field lines at the SAMA region for the satellite altitude $(660 \mathrm{~km})$ correspond to low values of the $L$ shell parameter $(L \sim 2)$. In order to see an effect of the geomagnetic storms in the SAMA region we defined a "calm day" when all $K p$ values in this day satisfy the condition $K p \leq 3$. In the same way, we defined a "day with a geomagnetic storm", when at least one value of the analysed day satisfies the condition $K p \geq 5$. The mean values for all the calm days and for all the geomagnetic storms days were computed. The difference of the electron flux for energies $E>0.270$ $\mathrm{MeV}$ between the mean field of geomagnetic storm days and the calm days is shown in Fig. $2 \mathrm{~b}$.As expected, there are only positive values, that correspond to electron fluxes larger during geomagnetic storm days than during calm days. There is a maximum enhancement in the order of $10^{4} \mathrm{~cm}^{-2} \mathrm{~s}^{-1} \mathrm{sr}^{-1}$ in the electron flux during geomagnetic storms in the SAMA region and a lower enhancement in the auroral zone (i.e. outer radiation belt). This enhancement during geomagnetic storms days is also observed for 


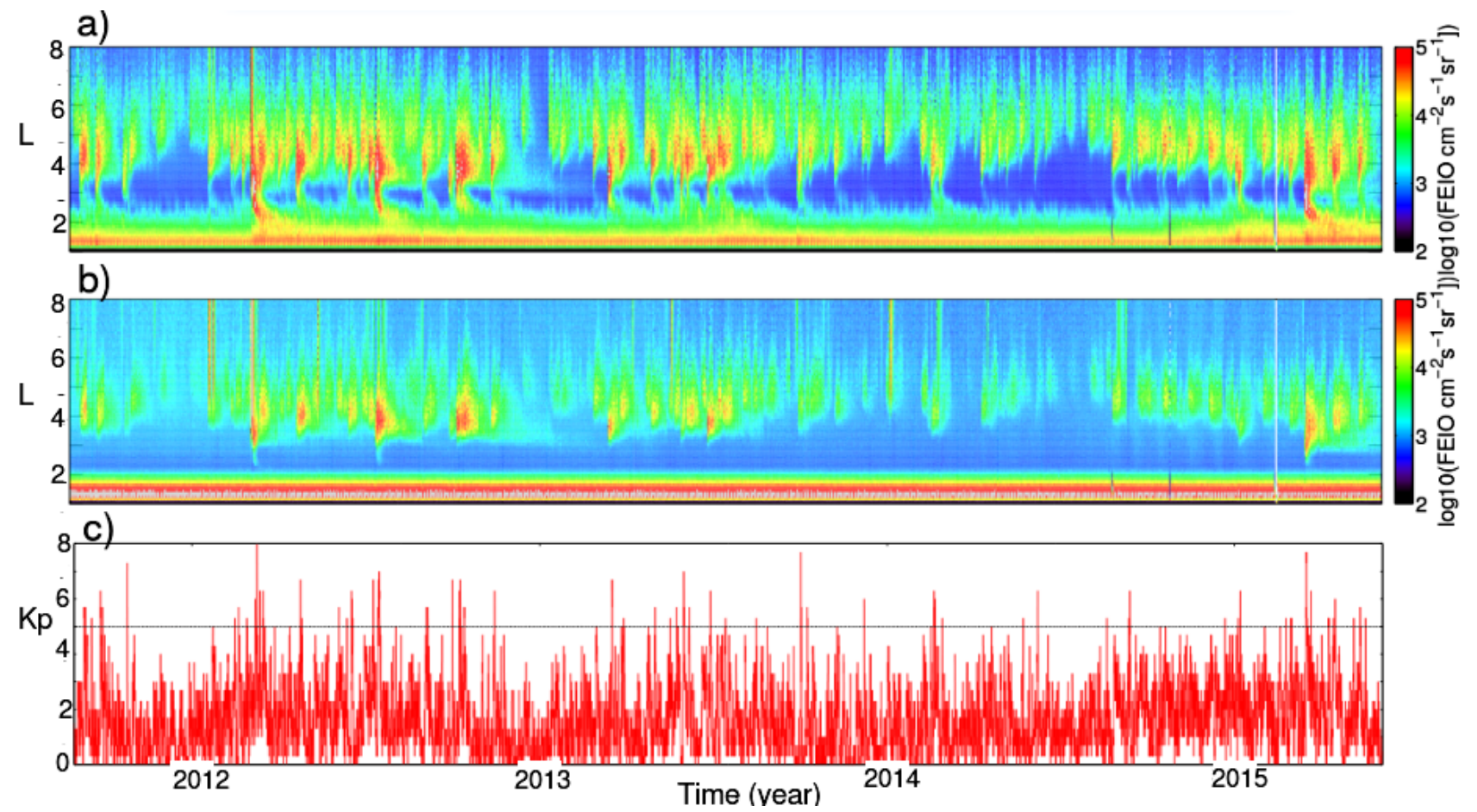

Figura 1: a) Daily averaged electron flux for energies $E>0.270 \mathrm{MeV}$ in function of time and $L$. b) Daily averaged electron flux for energies $E>0.802 \mathrm{MeV}$ in function of time and $L$. c) Geomagnetic index $K p$ in function of time, values that exceeds the black line $\left(K_{p}=5\right)$

the rest of the energy channels (not shown). From this Figure we conclude that the largest response of electron fluxes can be detected at high latitudes and in the SAMA region.

The tails of the distribution function (extreme events) of electron fluxes in the outer radiation belt and the SAMA are studied using the same statistical tool, which is based on the extreme value theory (see Section 2).

\subsection{OUTER RADIATION BELT REGION}

In the outer radiation belt, the POT method is applied to daily averaged electron fluxes.Then, the data are grouped in accordance with the $L$ parameter between $L=1-8$, in bins with sizes $\Delta L=0.25$. The threshold was defined for each energy channel and for each $L$ value by the 90th percentile of the daily averaged electron flux, considering the full analysed range time. The extreme values series are reconstructed for
7 energy channels in the range of $0.270 \mathrm{MeV}$ to $0.802 \mathrm{MeV}$ for the core of the outer radiation belt (i.e. $L=3.5-5.0$ ). The daily averaged electron flux in the inner edge and in the outer edge of the core of the outer radiation belt (i.e. $L=3.5-3.75$ and $L=4.5-4.75$ respectively) for energies $E>0.270 \mathrm{MeV}$ are shown in Fig. 3a,b.

The values that exceed the threshold in both panels of Fig. 3 are generally associated with intense geomagnetic storms $\left(K_{p}>5\right)$ as shown in Fig. 1.In the inner edge of the outer radiation belt (Fig. 3a), the extreme events are well defined as sudden increases in the electron flux. The extreme events can reach electron flux values of $1.4 \times 10^{5} \mathrm{~cm}^{-2} \mathrm{~s}^{-1} \mathrm{sr}^{-1}$. In the core of the outer radiation belt (Fig. $3 \mathrm{~b}$ ), these increases are not so well defined and the maximum of electron flux reach values of $5 \times 10^{4} \mathrm{~cm}^{-2} \mathrm{~s}^{-1} \mathrm{sr}^{-1}$ for the most intense geomagnetic storms. On the other hand, the 90th percentile threshold value is larger in the inner edge than in the 


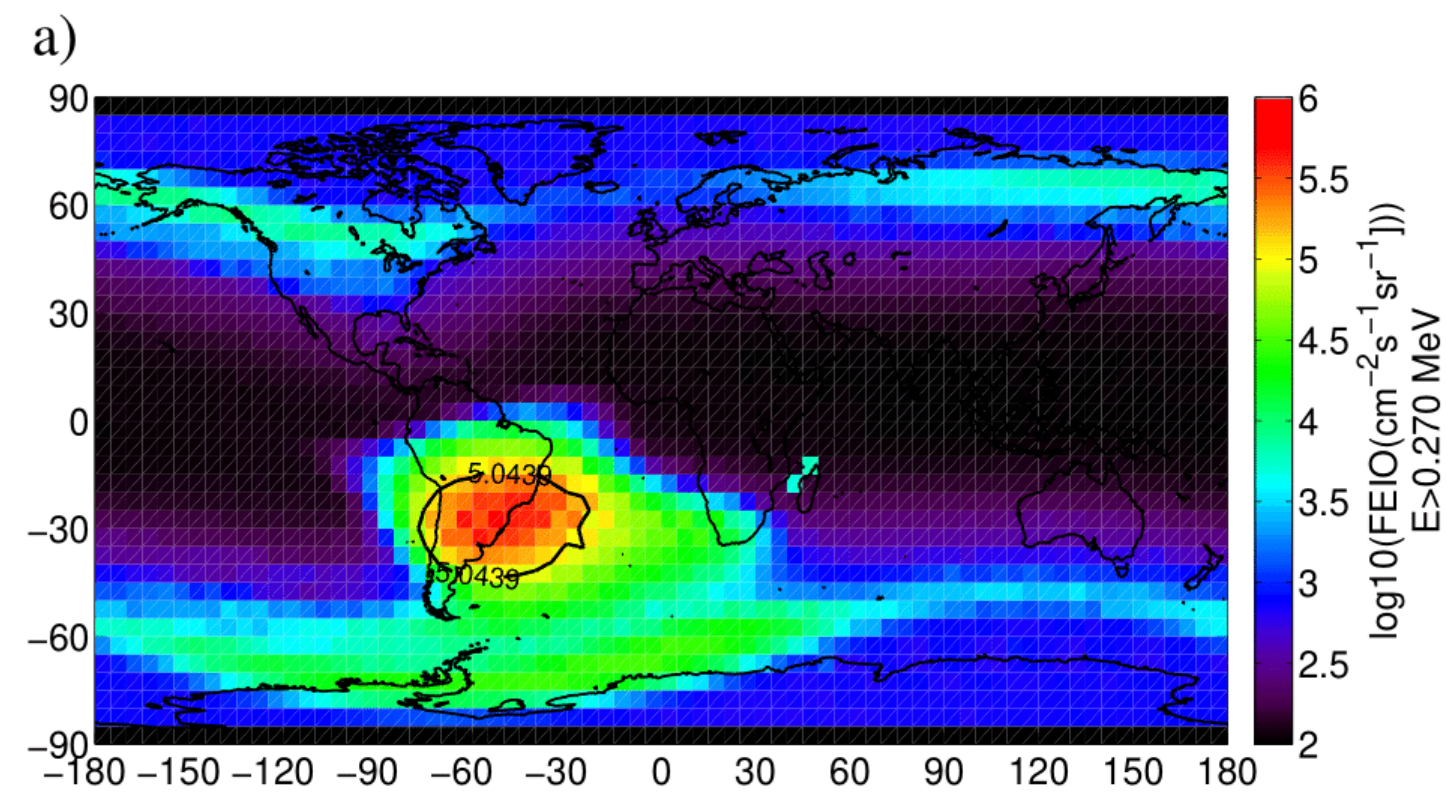

b)

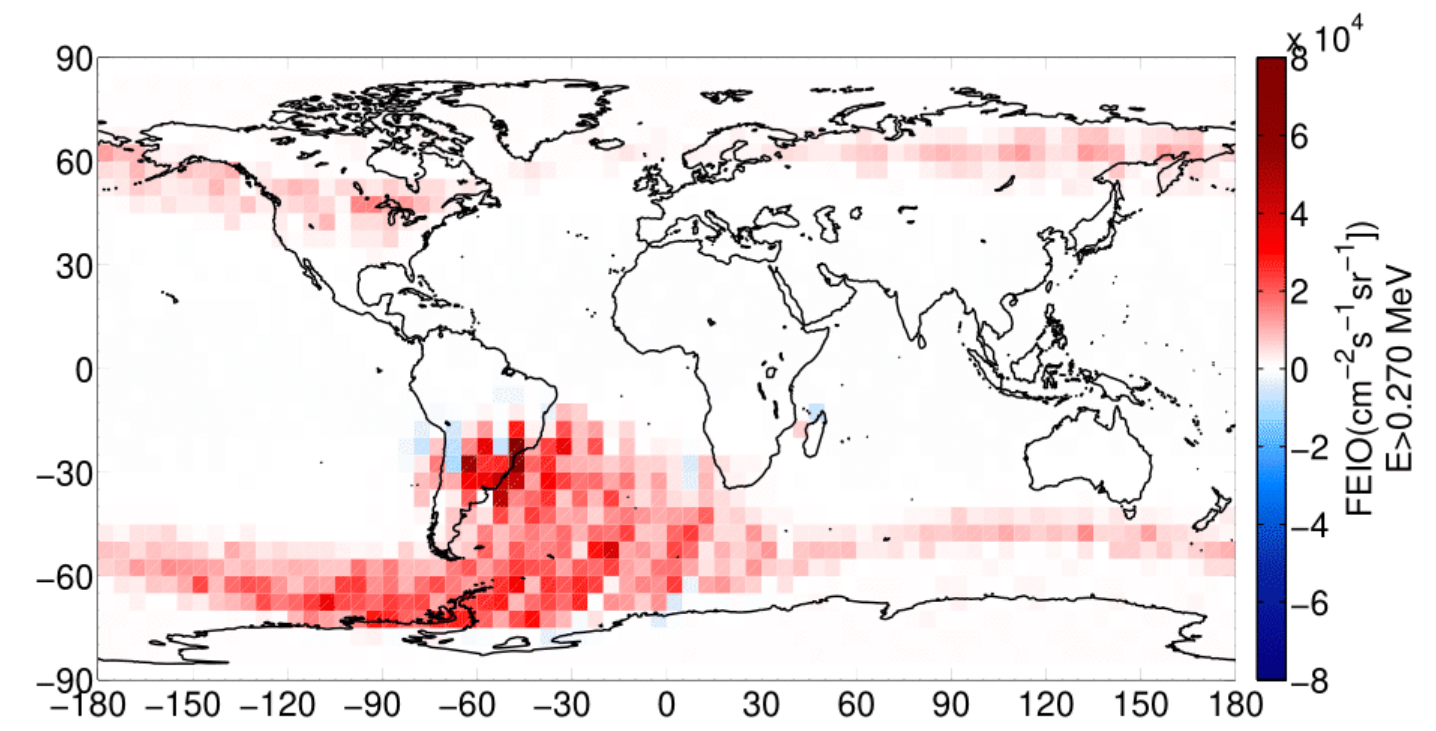

Figura 2: a) Mean field of electron flux $(E>0.270 \mathrm{MeV}$ ) from August/2011 to June/2015 at $\sim 660 \mathrm{~km}$ altitude (shaded). The SAMA is defined as the geographical region where the electron flux is higher than the 98th percentile value (contour). b) Electron flux difference between the mean value of 2011-2015 during geomagnetic storm days $(K p \geq 5)$ and the mean value during calm days $(K p \leq 3)$. 

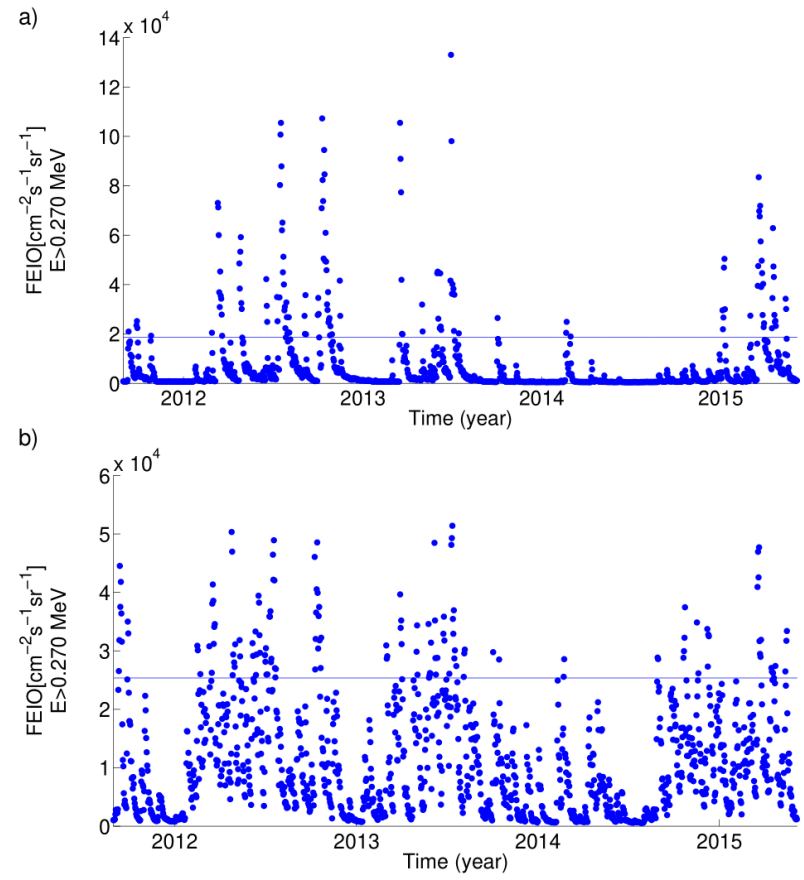

Figura 3: Scatterplot of daily averaged electron flux (dots) and 90th percentile value as threshold (line) between August/2011 to June/2015 for $E>0.270 \mathrm{MeV}$ at, a) $L=3.5-3.75$ corresponding to the inner edge of the outer radiation belt and b) $L=4.5-4.75$ corresponding to the core of the outer radiation belt.

core of the outer radiation belt.The electron flux variation over time is almost the same for the rest of the energy channels at a fixed $L$ bin value (not shown). The most important difference is that the magnitude of the flux is lower while increasing the energy.

\subsection{SAMA REGION}

In the SAMA region, the POT method is applied to the daily averaged electron fluxes that fill the core of the SAMA (i.e. all the data points inside the contour shown in Fig. 2). In this case, the SPE events were also removed as described before and we used the percentile $90 \%$ of the daily averaged electron flux in the core of the SAMA as the threshold.
Figure 4 shows the scatterplot of the daily averaged electron flux for two energy channels in the core of the SAMA. The horizontal line represents the threshold value. As expected, the figure shows, for both energies, sudden increases in the electron flux as in Fig. 3, associated with geomagnetic storms. Furthermore, the magnitude of the electron flux in the SAMA is one order magnitude larger than in the outer radiation belt, as shown in Fig. 2a (i.e., $10^{4}$ $\mathrm{cm}^{-2} \mathrm{~s}^{-1} \mathrm{sr}^{-1}$ in the outer radiation belt and $10^{5} \mathrm{~cm}^{-2} \mathrm{~s}^{-1} \mathrm{sr}^{-1}$ the SAMA region). Due to the proton flux contamination in the SAMA for the higher energy ranges, we focus the analysis to the extreme events in the lower energy channels (i.e. $0.249 \mathrm{MeV}, 0.270 \mathrm{MeV}$ and $0.299 \mathrm{MeV}$ ).

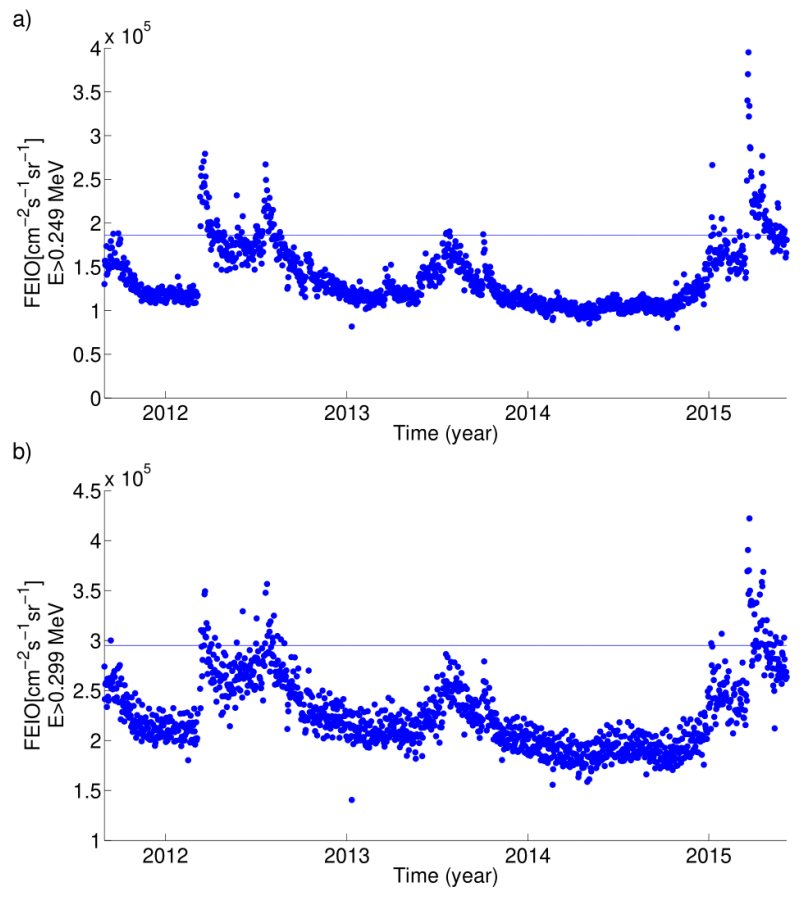

Figura 4: Scatterplot of daily averaged electron flux (dots) and 90th percentile value as threshold (line) between August/2011 to June/2015 at the SAMA region, for a) energies $E>0.249 \mathrm{MeV}$ and b) energies $E>0.299 \mathrm{MeV}$.

\section{RESULTS}

The daily averaged electron flux for 7 energy channels between $0.270 \mathrm{MeV}$ and $0.802 \mathrm{MeV}$ and in the range $L=3.5-5.0$ were reconstructed 
for the study of the outer radiation belt. For the SAMA we limit the analysis to its core defined in Fig. 2, and for energies between $0.249 \mathrm{MeV}$ and $0.299 \mathrm{MeV}$. In both cases we used the values that exceed the threshold $u$ defined as the 90th percentile as mentioned in Section 3. Then, we applied the maximum likelihood method to find the free parameters of the Generalised Pareto cumulative distribution function of Equation 1.

The cumulative distribution function of the electron fluxes from observations and the associated fitted GP function for the outer radiation belt are shown in Fig. 5 . It can be seen that this theoretical function applied to the tail (extreme cases) of distribution functions (shown in dashed line) well describe the observations (shown with circles). The distributions of the electron fluxes at $L=3.5-3.75$ are shown in Fig. 5a. The observed fluxes at any given energy cover over 1 order of magnitude. The largest observed fluxes cover over one order of magnitude, ranging from $5 \times 10^{4} \mathrm{~cm}^{-2} \mathrm{~s}^{-1} \mathrm{sr}^{-1}$ at $\mathrm{E}>0.802 \mathrm{MeV}$ to $1 \times 10^{5} \mathrm{~cm}^{-2} \mathrm{~s}^{-1} \mathrm{sr}^{-1}$ at $E>0.270 \mathrm{MeV}$. A similar plot for different $L$ shell parameters are shown in Figs. 5b-g. The largest observed fluxes increase for $L=3.75-4.0$ and then starts to decrease while increasing $L$. Moreover, the observed flux for any giver energy cover over a smaller range while increasing the $L$ parameter.

The estimated shapes parameters $k$ for the outer radiation belt are shown in Table 1 . In general, for $L \geq 4$ there are more cases with negative $k$ values, suggesting that the distribution function of the extreme cases is finite. However, only in some cases, the $\mathrm{k}$ error bar provides negative values with $95 \%$ confidence (shown in bold). In the inner edge of the outer radiation belt $(3.5<$ $L<3.75)$ both signs of $\mathrm{k}$ are found without statistical significance. We also notice that for all the range of $\mathrm{L}$ and energies a significantly positive value of $\mathrm{k}$ is not reported. The negative shape parameter found in $L=4.5-4.75$ is found to be in accordance with the results of Meredith et al. (2017).
For the cases where $k$ is significantly negative, the return values $X_{N}$ for 10,50 and 100 years were computed using the Equation 2. Fig. 6 shows the largest expected electron that is likely to be observed over the three different periods of time at $L=4.5-4.75$ and for different energy channels. The 1 in 10 year electron flux shows a general decreasing trend with energy ranging from $3.5 \times 10^{4} \mathrm{~cm}^{-2} \mathrm{~s}^{-1} \mathrm{sr}^{-1}$ for $E>0.249 \mathrm{MeV}$ to $1.5 \times 10^{4} \mathrm{~cm}^{-2} \mathrm{~s}^{-1} \mathrm{sr}^{-1}$ for $E>0.802 \mathrm{MeV}$. The same behaviour is observed for the 1 in 50 and 1 in 100 year event. Furthermore, for all the energy channels the return value is larger as the waiting time increases, although the behaviour is not linear. For example, for $E>0.249 \mathrm{MeV}$ the expected electron flux between 50 and 10 years differs in $1 \times 10^{4}$ meanwhile between 100 and 50 this difference is smaller, $X_{100}-X_{50} \approx$ $0.25 \times 10^{4}$.

The shape parameter $(k)$ values for energies $E>$ $0.249 \mathrm{MeV}$ and $E>0.270 \mathrm{MeV}$ in the SAMA are marginally negative, but the $95 \%$ interval confidence makes $k$ both positive and negative. Moreover, the shape parameter for $E>0.299$ $\mathrm{MeV}$ is marginally positive with an error bar that also makes $k$ both positive and negative. Thus, it is not possible to infer the behaviour of the tail of extreme electron fluxes in the SAMA.

\section{SUMMARY AND CONCLUSIONS}

The energetic electrons fluxes in the outer radiation belt are the source of many of the technological hazards for satellites in any Earth orbit. Depending on the energy of these electron, they can produce different damages to the spacecrafts. Especially during a geomagnetic storm, the electron fluxes can increase dramatically. Furthermore, as the SAMA is a region where the magnitude of the geomagnetic field is weaker, the electron fluxes over this region reach lower altitudes. The aim of this work is to study the extreme electron fluxes in the outer radiation belt and a special emphasis in the SAMA region. We studied the tails of the distribution function using the extreme value theory, in particular we used 

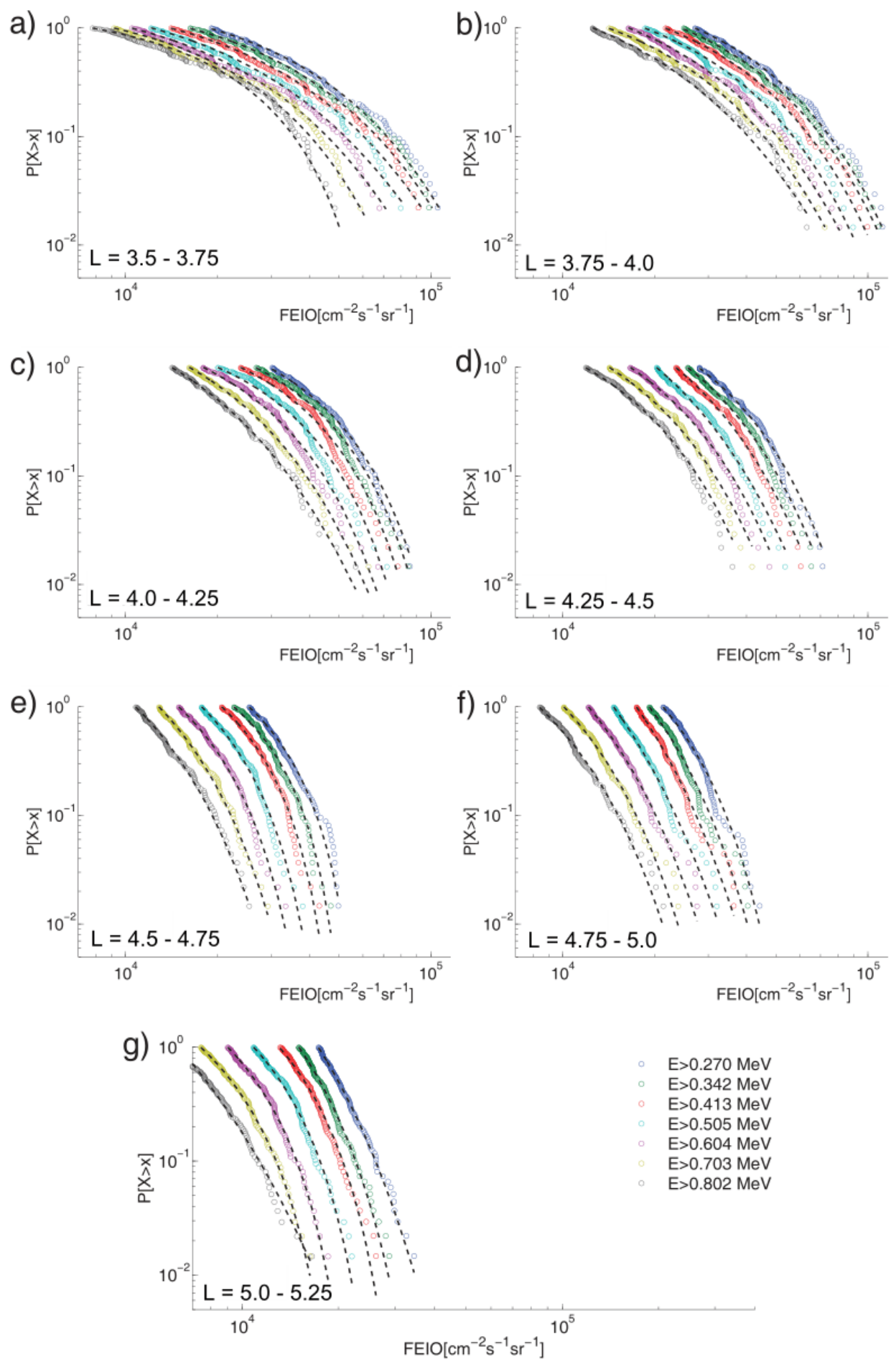

Figura 5: Extreme value analysis for seven energy channels. Cumulative distribution from observations (circles) and associated fitted GP functions (dashed) for a) $L=3.5-3.75$, b) $L=3.75-4.0$, c) $L=4.0-4.25$, d) $L=4.25-4.5$, e) $L=4.5-4.75$, f) $L=4.75-5.0$ and g) $L=5.0-5.25$. 


\begin{tabular}{lccccccc}
\hline Energy & \multicolumn{7}{c}{ L range } \\
\cline { 2 - 8 }$(\mathrm{MeV})$ & $3.5-3.75$ & $3.75-4.0$ & $4.0-4.25$ & $4.25-4.5$ & $4.5-4.75$ & $4.75-5.0$ & $5.0-5.25$ \\
\hline 0.270 & -0.005 & -0.047 & $\mathbf{- 0 . 1 7 0}$ & -0.094 & $\mathbf{- 0 . 0 2 6}$ & -0.012 & -0.140 \\
0.342 & 0.017 & -0.037 & $\mathbf{- 0 . 1 6 0}$ & -0.056 & $\mathbf{- 0 . 0 2 9}$ & 0.062 & -0.100 \\
0.413 & 0.044 & 0.047 & $\mathbf{- 0 . 1 7 0}$ & -0.031 & $\mathbf{- 0 . 2 5 0}$ & -0.008 & $\mathbf{- 0 . 1 9 0}$ \\
0.505 & 0.080 & -0.040 & $\mathbf{- 0 . 1 7 0}$ & 0.0002 & $\mathbf{- 0 . 1 9 0}$ & 0.009 & $\mathbf{- 0 . 1 9 0}$ \\
0.604 & 0.041 & 0.013 & -0.110 & -0.056 & $\mathbf{- 0 . 1 7 0}$ & 0.024 & $\mathbf{- 0 . 2 0 0}$ \\
0.703 & -0.056 & 0.005 & -0.021 & -0.050 & -0.082 & -0.058 & $\mathbf{- 0 . 1 9 0}$ \\
0.802 & $\mathbf{- 0 . 2 2 0}$ & 0.065 & 0.026 & -0.065 & -0.054 & -0.053 & 0.063 \\
\hline
\end{tabular}

Tabla I: Estimated shape parameter $(k)$ values for the outer radiation belt Region. Bold values indicate values with $95 \%$ confidence

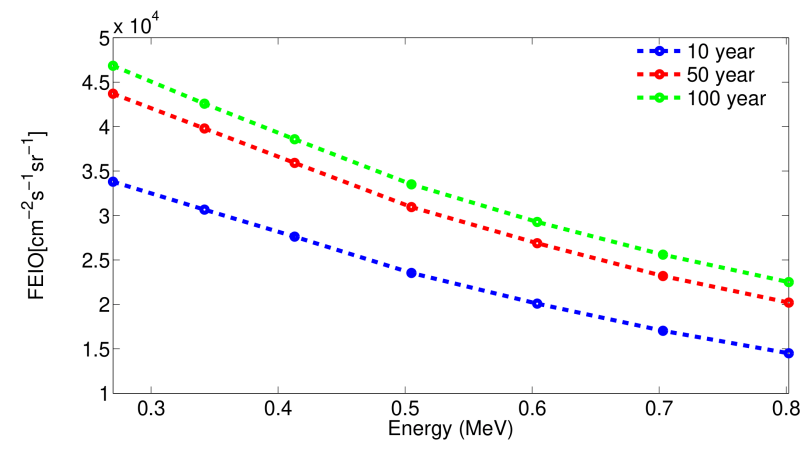

Figura 6: Return values for 10, 50 and 100 years for $L=4.5-4.75$ in the range of electron energies between $E>0.249 \mathrm{MeV}$ to $E>0.802 \mathrm{MeV}$.

the peaks over threshold method. We used data from the ICARE-NG particle detector on board the Argentinean SAC-D spacecraft, which provides data of the electron fluxes in different energies channels (between $E>0.240 \mathrm{MeV}$ and $E>0.802 \mathrm{MeV}$ ) and in a wide range of $L$ values. Some preliminary results are present in Lanabere and Dasso (2018).

The peaks over threshold method was applied to the daily averaged electron flux in the outer radiation belt for different $L$ ranges. Also, the method was applied to the daily averaged electron flux inside the SAMA region. In both cases we defined the threshold as the 90th percentile of the full data set for each $L$ and energy value. Then, the maximum likelihood method was applied to estimate the shape parameter $(k)$ in the outer radiation belt and in the SAMA region for different energy channels between $E>0.240 \mathrm{MeV}$ and $E>0.802 \mathrm{MeV}$.

In this work we found that negative shape parameters dominate in the outer radiation belt for $L>4$. Furthermore, in some cases we found negative shape parameter with $95 \%$ confidence but no statistically significant positive shape parameters.

In particular, at $L=4.5-4.75$ these results are consistent with that found in Meredith et al. (2017) and in O'Brien et al. (2007). Where Meredith et al. (2017) found significant negative shape parameter $(k<0)$ centered at $L=4.5$ for energies between $0.69 \mathrm{MeV}$ and $2.05 \mathrm{MeV}$ and O'Brien et al. (2007) found evidence of negative $k$ values for energies from $100 \mathrm{keV}$ to some $\mathrm{MeV}$ throughout the outer radiation belt $L=2-8$. Also, for lower energies, Meredith et al. (2016) found that in the region $L=4-8$ and with $E>30 \mathrm{keV} \mathrm{k}$ is negative. Although, Meredith et al. (2016) found that the shape parameter for $E>100 \mathrm{keV}$ and $E>300 \mathrm{keV}$ are positive.

In this case, the electron flux return values for 10, 50 and 100 years were computed. The return values for the three cases shows a general decreasing trend with energy. Furthermore, for all the energy channels the return value is larger as the waiting time increases, although the 
behaviour is not linear.

However, in the SAMA region the error bar of the shape parameter give negative and positive values, so it is not possible to infer the behaviour of the tail of the extreme fluxes.

These results are important to understand the environment encountered by satellites passing through the outer radiation belt, in particular, the extremes of this environment to be able to better protect space assets operating in this region and the impact on the resulting life expectancy of the satellite. We advise that our flux limits published here are not used for decision making since our analysis is only for academic purposes, and it is limited in time. A deeper and more conclusive analysis requires a larger data set that covers multiple solar cycles. Although, the analysis was applied to a short data base obtained from ICARE-NG on board Argentinean satellite SAC-D, we found consistent results with the results of other authors. Despite this instrument on board SAC-D stopped operating in June 2015, the same kind of particle detector (ICARE-NG) was on board Jason-2 (2008-2019) and it is at present on board Jason-3 launched 2016. So, we expect in a future to include this data in order to extend the data set in order to have more statistic and to cover a full solar cycle.

The flux limits found in this work correspond to the studied phase of the solar cycle and may not represent the absolute maximum flux, since our data covers the maximum phase of solar cycle 24, meanwhile it is well known that the maximum electron flux is observed during the declining phase (Miyoshi and Kataoka, 2011).

ACKNOWLEDGEMENTS: The authors thank CONAE (Comisión Nacional de Actividades Espaciales, in spanish) for providing the data used for this work. The authors acknowledge partial support from the Argentinian grants UBACyT (UBA) and PIDDEF 2014/8
(Ministerio de Defensa, Argentina).

\section{REFERENCIAS}

Abdu, M. A., I. S. Batista, L. R. Piazza, and O. Massambani, 1981: Magnetic storm associated enhanced particle precipitation in the South Atlantic Anomaly: Evidence from VLF phase measurements. Journal of Geophysical Research: Space Physics, 86 (A9), 7533-7542, doi: 10.1029/JA086iA09p07533

Bartels, J., 1949: The standardized index ks and the planetary index kp. IATME Bull, 12b, 97

Boscher, D., and Coauthors, 2011: In Flight Measurements of Radiation Environment on Board the French Satellite JASON-2. Nuclear Science, IEEE Transactions on, 58, 916-922

Boscher, D., and Coauthors, 2014: In-Flight Measurements of Radiation Environment on Board the Argentinean Satellite SAC-D. IEEE Transactions on Nuclear Science, 61 (6), 3395-3400, doi:10.1109/TNS.2014.2365212

Caraballo, R., L. Sánchez Bertucci, and G. Tancredi, 2013: Geomagnetically induced currents in the Uruguayan high-voltage power grid. Geophysical Journal International, 195, 844-853, doi:10.1093/gji/ggt293

Claudepierre, S. G., and Coauthors, 2015: A background correction algorithm for van allen probes mageis electron flux measurements. Journal of Geophysical Research: Space Physics, 120 (7), 5703-5727, doi:10.1002/2015JA021171

Coles, S., 2013: An Introduction to Statistical Modeling of Extreme Values. Springer Series in Statistics, Springer London

de Villiers, J. S., R. J. Pirjola, and P. J. Cilliers, 2016: Estimating ionospheric currents by inversion from ground-based geomagnetic data and calculating geoelectric fields for studies of geomagnetically induced currents. eos transactions american geophysical union, 68 (1), 154, doi:10.1186/s40623-016-0530-1

Elvidge, S., and M. J. Angling, 2018: Using Extreme Value Theory for Determining 
the Probability of Carrington-Like Solar Flares. Space Weather, 16 (4), 417-421, doi:10.1002/2017SW001727

Heirtzler, J. R., J. H. Allen, and D. C. Wilkinson, 2002: Ever-present South Atlantic Anomaly damages spacecraft. Eos, Transactions American Geophysical Union, 83 (15), 165-169, doi: 10.1029/2002EO000105

Horne, R. B., M. M. Lam, and J. C. Green, 2009: Energetic electron precipitation from the outer radiation belt during geomagnetic storms. Geophysical Research Letters, 36 (19), doi: 10.1029/2009GL040236

Koons, H. C., 2001: Statistical analysis of extreme values in space science. Journal of Geophysical Research: Space Physics, 106 (A6), 10915-10921, doi:10.1029/2000JA000234.

Koons, H. C., and J. F. Fennell, 2006: Space weather effects on communications satellites. URSI Radio Science Bulletin, 2006 (316), 27-41, doi:10.23919/URSIRSB.2006.7909358.

Lanabere, V., and S. Dasso, 2018: Statistical analysis of extreme electron fluxes in the radiation belts. Proc. of Space Weather of the Heliosphere: Processes and Forecasts, IAU SymposiumUK, Cambridge University Press, 335, 128-131, doi:10.1017/S1743921317011000.

McIlwain, C. E., 1961: Coordinates for mapping the distribution of magnetically trapped particles. Journal of Geophysical Research, 66 (11), 3681-3691, doi:10.1029/JZ066i011p03681.

Meredith, N. P., R. B. Horne, J. D. Isles, and J. C. Green, 2016: Extreme energetic electron fluxes in low Earth orbit: Analysis of POES E ¿30, E ¿100, and E ¿300 keV electrons. Space Weather, 14 (2), 136-150, doi:10.1002/2015SW001348

Meredith, N. P., R. B. Horne, J. D. Isles, and J. V. Rodriguez, 2015: Extreme relativistic electron fluxes at geosynchronous orbit: Analysis of GOES E i2 MeV electrons. Space Weather, 13 (3), 170-184, doi:10.1002/2014SW001143.
Meredith, N. P., R. B. Horne, I. Sandberg, C. Papadimitriou, and H. D. R. Evans, 2017: Extreme relativistic electron fluxes in the Earth's outer radiation belt: Analysis of INTEGRAL IREM data. Space Weather, 15 (7), 917-933, doi:10.1002/2017SW001651

Miyoshi, Y., and R. Kataoka, 2011: Solar cycle variations of outer radiation belt and 447 its relationship to solar wind structure dependences. Journal of Atmospheric and Solar-448 Terrestrial Physics, 73 (1), 77-87, doi:10.1016/j.jastp.2010.09.031

Nishino, M., K. Makita, K. Yumoto, F. S. Rodrigues, N. J. Schuch, and M. A. Abdu, 2002: Unusual ionospheric absorption characterizing energetic electron precipitation into the South Atlantic Magnetic Anomaly. Earth, Planets and Space, 54 (9), 907-916, doi: 10.1186/BF03352438

O'Brien, T. P., J. F. Fennell, J. L. Roeder, and G. D. Reeves, 2007: Extreme electron fluxes in the outer zone. Space Weather, 5 (1), doi:10.1029/2006SW000240

Pickands, J., 1975: Statistical Inference Using Extreme Order Statistics. The Annals of Statistics, 3 (1), 119-131

Prölss, G., 2012: Physics of the Earth's Space Environment: An Introduction. Springer Berlin Heidelberg.

Re, M., and V. R. Barros, 2009: Extreme rainfalls in se south america. Climatic Change, 96 (1), 119-136, doi:10.1007/s10584-009-9619-x

Reeves, G. D., K. L. McAdams, R. H. W. Friedel, and T. P. O'Brien, 2003: Acceleration and loss of relativistic electrons during geomagnetic storms. Geophysical Research Letters, 30 (10), doi:10.1029/2002GL016513

Ruzmaikin, A., J. Feynman, and I. Jun, 2011: Distribution of extreme solar energetic proton fluxes. Journal of Atmospheric and Solar-Terrestrial Physics, 73, 300-307, doi:10.1016/j.jastp.2009.12.016

Sheldon, W. R., and J. R. Benbrook, 2004: The sink for outer belt electrons and the electrodynamics of the middle atmosphere. Advances in Space Research, 34, 1806-1810, 
doi:10.1016/j.asr.2003.06.033

Smirnov, A. G., and Coauthors, 2019: Electron intensity measurements by the cluster/rapid/ies instrument in earth's radiation belts and ring current. Space Weather, 17 (4), 553-566, doi:10.1029/2018SW001989

Tencer, B., and M. Rusticucci, 2012: Analysis of interdecadal variability of temperature extreme events in Argentina applying EVT. Atmósfera, 25, 327-337

Trivedi, N. B., B. M. Pathan, N. J. Schuch, M. Barreto, and L. G. Dutra, 2005: Geomagnetic phenomena in the South Atlantic anomaly region in Brazil. Advances in Space Research, 36, 2021-2024, doi:10.1016/j.asr.2004.09.020

Vampola, A. L., 1998: Measuring Energetic Electrons-What Works and What Doesn't. Washington DC American Geophysical Union Geophysical Monograph Series, 102, 339, doi: 10.1029/GM102p0339

Walt, M., 2005: Introduction to Geomagnetically Trapped Radiation. Cambridge Atmospheric and Space Science Series, Cambridge University Press

Wrenn, G. L., D. J. Rodgers, and K. A. Ryden, 2002: A solar cycle of spacecraft anomalies due to internal charging. Annales Geophysicae, 20, 953-956, doi:10.5194/angeo-20-953-2002

Xiong, Y., and Coauthors, 2015: Responses of relativistic electron fluxes in the outer radiation belt to geomagnetic storms. Journal of Geophysical Research: Space Physics, 120 (11), 9513-9523, doi:10.1002/2015JA021440 